\title{
Simulations of the evolution of the X-Ray properties of a young stellar population
}

\author{
M. Eracleous ${ }^{1}$, M. S. Sipior ${ }^{1} \dagger$, S. Sigurdsson ${ }^{1}$ and H. Flohic ${ }^{1}$ \\ ${ }^{1}$ Department of Astronomy \& Astrophysics, The Pennsylvania State University, 525 Davey \\ Lab, University Park, PA 16802, USA
}

\begin{abstract}
We present an X-ray binary population synthesis model, and use it to simulate the evolution of X-ray binaries formed in a burst of star formation of duration $20 \mathrm{Myr}$ and starformation rate $10 \mathrm{M}_{\odot} \mathrm{yr}^{-1}$. We find that the X-ray luminous phase persists long after the star-formation episode has ended. The $2-10 \mathrm{keV}$ X-ray luminosity can reach values of $10^{40}$ $10^{41} \mathrm{erg} \mathrm{s}^{-1}$, comparable to those of some Seyfert galaxies. These models are directly applicable to starburst galaxies as well as LINERs powered by vigorous star formation.
\end{abstract}

\section{Introduction}

In our quest for the power source of LINERs we have found, through observations with the Chandra X-ray observatory, that a significant fraction of objects host clusters of luminous $\left(L_{\mathrm{x}} \sim 10^{38} \mathrm{erg} \mathrm{s}^{-1}\right) \mathrm{X}$-ray binaries in their nuclei. The X-ray binary luminosity functions are flatter than those of elliptical galaxies and resemble those of star-forming and starburst galaxies (Eracleous et al. 2002; Flohic et al. 2004). This suggests the presence of high-mass X-ray binaries formed in a recent episode of star-formation. To understand the origin and properties of these X-ray binaries and to relate them to starformation activity in the LINER nuclei, we have constructed binary population synthesis models and used them to predict the integrated X-ray properties of the population.

\section{Methodology}

We used a modified version of the code of Pols \& Marinus (1994) to follow for 2 Gyr the evolution of binaries from a $20 \mathrm{Myr}$ star-formation episode with $S F R=10 \mathrm{M}_{\odot} \mathrm{yr}^{-1}$. Our modifications include: a mapping of progenitor to black hole mass based on recent collapse simulations (Fryer 1999), an up-to-date supernova kick distribution, semi-analytic mass transfer prescriptions at different evolutionary stages (Hurley et al. 2002), magnetic braking, and accretion-disk instabilities and duty cycles. Details are given in Sipior et al. (2004). We explored two binary mass fraction distributions (flat and low-biased) and two IMFs (Salpeter and Miller-Scalo).

\section{Results and Discussion}

Our primary result, the evolution of the integrated X-ray luminosity luminosity of the population (assuming a low-skewed mass ratio distribution and a Salpeter IMF), is shown in Figure 1. This result is not sensitive to the assumed mass ratio distribution or IMF. There is a prolonged X-ray luminous phase, which may last $100 \mathrm{Myr}$ past the end of star formation. During this phase, the X-ray luminosity is sustained by successive generations

$\dagger$ Present address: Astronomical Institute "Anton Pannekoek" and Section Computational Science, University of Amsterdam, Kruislaan 403, 1098 SJ Amsterdam, Netherlands. 


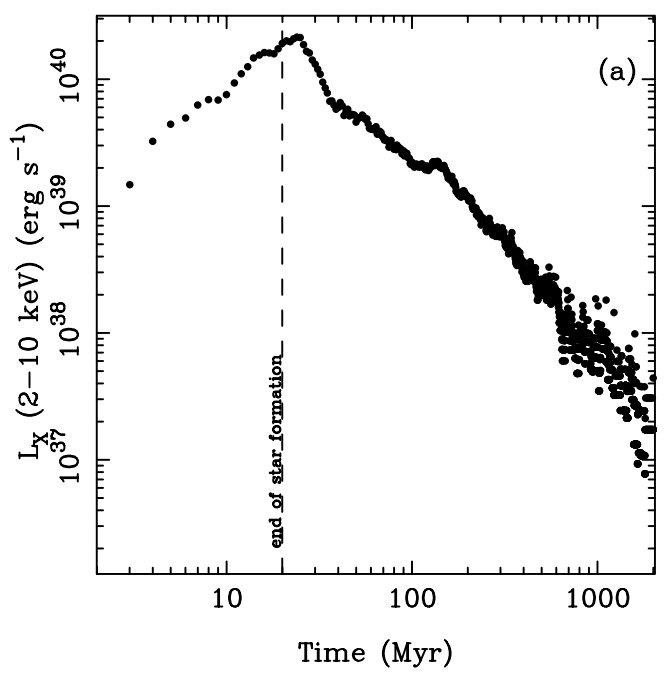

Figure 1. The evolution of the total $2-10 \mathrm{keV}$ X-ray luminosity of a simulated population, such as that described in $\S 2$. For this simulation we assumed a Salpeter IMF with a low-skewed binary mass ratio distribution.

of X-ray binaries, which rise and fall at different times, depending on the masses of the donnor stars. At its peak, the X-ray luminosity is $3 \times 10^{40} \mathrm{erg} \mathrm{s}^{-1}$, and should scale with SFR. The power-law index of the cumulative luminosity function, $N(>L) \propto L^{-\beta}$, evolves from $\beta \approx 0.8$ at $10 \mathrm{Myr}$ to $\beta \approx 3$ at $100 \mathrm{Myr}$. The models reproduce the empirical relation between SFR and X-ray luminosity, $S F R=2\left(L_{\mathrm{x}} / 10^{40} \mathrm{erg} \mathrm{s}^{-1}\right) \mathrm{M}_{\odot} \mathrm{yr}^{-1}$ (e.g., Ranalli et al. 2003), to better than a factor of 2 . They also reproduce the slope of the cumulative luminosity function of star-forming galaxies (e.g., Colbert et al. 2004) and its steepening with time. At late times, after the demise of OB stars (post-starburst phase), the late B and post-AGB stars provide enough ionizing photons to power line emission with luminosities and line ratios as observed in LINERs (Binette et al. 1994; Magris et al. 2003). Such objects would have X-ray spectra and luminosities similar to weak AGNs, even though they are powered by star formation.

\section{Acknowledgements}

This work was supported by NASA through grants GO0-1152A,B and AR4-5010A from the Smithsonian Astrophysical Observatory. SS acknowledges support from NSF grant PHY-0203046 and from the Center for Gravitational Wave Physics, which is supported by the NSF under cooperative agreement PHY 01-14375.

\section{References}

Binette, L., Magris, C. G., Stasińska, G., \& Bruzual, G. A. 1994, A\&A, 292, 13

Colbert, E. J. M., et al. 2004, ApJ, 602, 231

Eracleous, M., Shields, J. C., Chartas, G., \& Moran, E. C. 2002, ApJ, 565, 108

Flohic, H., et al. 2004, ApJ, 2004, in preparation

Fryer, C. L. 1999, ApJ, 522, 413

Hurley, J. R., Tout, C. A., \& Pols, O. R. 2002, MNRAS, 329, 897

Magris, C. G., Binette, L., \& Bruzual, G. A. 2003, ApJS, 149, 313

Pols, O. R., \& Marinus, M. 1994, A\&A, 288, 475

Ranalli, P., Comastri, A., \& Setti, G. 2003, A\&A, 399, 39

Sipior, M. S., Eracleous, M., \& Sigurdsson, S. 2004, ApJ, submitted (astro-ph/0308077) 